\title{
The Cyclotron and its Applications*
}

\author{
By Prof. J. Chadwick, F.R.S.
}

$\mathrm{I}^{\mathrm{N}}$ the early work on the artificial disintegration of elements by $\alpha$-particles, it was thought that particles of very high energy were necessary in order to penetrate the nuclei of even the lighter elements, and there appeared little, if any, hope of ever extending these experiments to the heavy elements.

The application of quantum mechanics to the problem of the penetration of potential barriers, however, indicated that particles of comparatively low energy should have a small, but not negligible, probability of entering the nuclei of the light elements. Now the small chance of penetration can be compensated by using large numbers of particles, and Cockcroft and Walton realized that this might make it possible to observe disintegrations with particles of energy of only a few hundred kilovolts. They carried out the first successful experiment of this kind in 1932, when they observed the disintegration of lithium under the bombardment of protons. Using potentials up to $600 \mathrm{kv}$. to accelerate the ions obtained from a hydrogen discharge tube, they were able to disintegrate several of the lighter elements. They were, however, unable to produce disintegrations in the heavier elements. The reason for this limitation is that the height of the nuclear barrier opposing the entry of the proton increases with the atomic number of the element. In order to disintegrate the heavy elements, we must use particles of much greater energy than those used by Cockcroft and Walton. In fact, we require accelerating voltages of some millions of volts instead of some hundred thousands.

During the last ten years, several methods have been developed for accelerating ions-protons, deuterons or $\alpha$-particles - to the very high energies necessary for these experiments on the transmutation of elements. The methods may be divided into two classes, the direct methods, in which a high voltage is developed and applied to a discharge tube, and the indirect methods. The direct methods have some serious disadvantages. First, they are limited to what for the present purpose are moderate voltages. Experimental difficulties increase rapidly as the voltage is increased, and so far the highest voltage which has been used directly for the acceleration of ions is two million volts. Secondly, high-voltage

* From the Friday evening discourse given at the Royal Institution on May 27. outfits need much space. The cost of installing an outfit operating at, say, 5 million volts, would be very high for the building alone, for few, if any, physical laboratories possess the large and lofty hall which is necessary to house such an apparatus.

In the indirect methods of accelerating ions, a relatively small voltage is applied many times in succession, increasing the energy of the particles in a series of small steps.

The most successful of these indirect methods is that used in the cyclotron, or magnetic resonance accelerator, which has been developed by Prof. E. O. Lawrence and his collaborators in the University of California.

Suppose a particle of mass $m$ and charge $e$ moves with velocity $v$ in a plane at right angles to a magnetic field $H$. The particle will move in a circular path of radius $\rho$ where $\rho$ is given by

$$
m v^{2} / \rho=H e v
$$

The time taken to complete a revolution is

$$
\frac{2 \pi p}{v}=2 \pi \cdot \frac{m}{e} \cdot \frac{\mathbf{l}}{H}
$$

The time of revolution does not depend on the radius of the path or on the velocity of the particle. For ions of the same $e / m$, the time of revolution will always be the same in the same field.

Now suppose that we have in the field two electrodes between which we can apply an alternating voltage, $V$. Let an ion start with very small velocity at some point between the electrodes. If the top electrode happens to be positive at that moment, the ion will be driven downwards. It will travel in a semi-circular path and return to the gap between the electrodes. Its energy will be $e V$. If at this moment the top electrode is negative, it will be driven upwards, acquiring another increment of energy $\mathrm{eV}$. Since the particle always takes the same time to go round the semi-circle and return to the gap, it should be possible by adjusting the frequency of the A.C. voltage and the strength of the magnetic field to keep in step with the particle, that is, to give the particle an impulse every time it crosses the electrodes.

Whether this method is possible in practice will depend first on whether one can produce oscillating voltages of the required frequency. Let us calculate what frequency is required for a proton. 
The time of rotation is $2 \pi \cdot \frac{m}{e} \cdot \frac{1}{H}$, and for a proton is equal to $6.57 \times 10^{-4} / H$ sec. The final velocity of the proton is proportional to $H \rho$, where $\rho$ is the radius of its final orbit. Since it is desirable to keep the dimensions of the apparatus-and especially the dimensions of the magnet-as small as possible, $H$ must be large. With good mild steel, $H$ may be as much as 19,000-20,000 gauss. Let us take $H$ equal to 15,000 gauss as a readily attainable strength of field. Then the time of rotation is $4 \cdot 38 \times 10^{-8} \mathrm{sec}$. The frequency of the alternating field must therefore be $2.28 \times 10^{-7}$

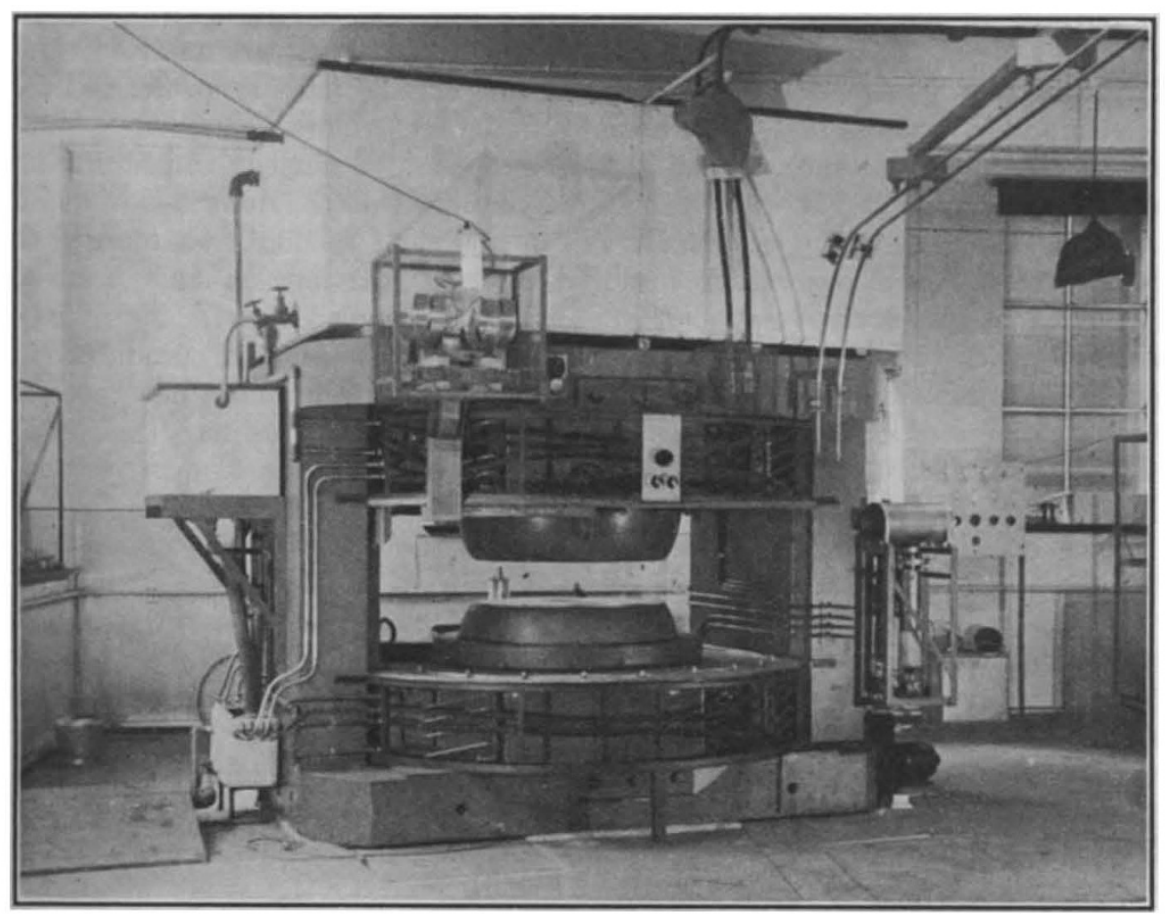

Magnet OF THE Lrverpool CyCLOTRON

THE PHOTOGRAPH, TAKEN AT AN EARLY STAGE IN THE CONSTRUCTION OF THE CYCLOTRON, SHOWS THE MAGNET ASSEMBIY WITH ITS WATER SUPPLIES, FTC., BUT WITHOUT THE VACUUM CHAMBER AND ITS AUXILIARIES

cycles per sec., corresponding to a wave-length of about 13 metres.

As the time of rotation is proportional to $\mathrm{m} / \mathrm{e}$, the frequencies of the oscillations required to accelerate deuterons or helium ions ( $\alpha$-particles) under the same conditions will be half as great, corresponding to a wave-length of 26 metres.

We have now to calculate what speeds the particles can acquire in this way. The maximum velocity is determined by $H \rho$, that is, it depends not only on the strength of the field but also on its extent. In the magnet at Liverpool the diameters of the pole faces are $94 \mathrm{~cm}$., and we can allow $P$ to be about $38 \mathrm{~cm}$. With $H$ equal to 15,000 gauss, the proton would reach a speed of $5.4 \times 10^{9}$ cm./sec., or an energy of 15 million volts. Under corresponding conditions, deuterons would acquire an energy of 8 million volts.

At first sight it seems that exceedingly high energies might be reached, for it is quite feasible to increase $H$ to 20,000 gauss and $\rho$ to $60 \mathrm{~cm}$. or more. A proton of $H_{p}$ equal to $1.2 \times 10^{6}$ would have an energy of about 60 million volts. However, a serious difficulty appears when the protons move at a speed corresponding to more than about 10 million volts (deuterons at about 15 million volts). This difficulty arises from the relativity increase of mass of a particle with its speed. The relation $H p=m v / e$ is no longer sufficiently precise, and we must write

$$
H_{\rho}=\frac{m}{e} \cdot \frac{v}{\sqrt{1-v^{2} / c^{2}}} .
$$

The particle will not keep in step with the electrical oscillations, that is, there will not be resonance. One could try to obtain exact resonance by adjusting the magnetic field so that it increases gradually as $\rho$ in. creases, but this introduces another defect which may result in complete loss of the ion beam. It may prove possible to find a suitable device to overcome these troubles, but at the present moment it seems that limits are set to the energies which it is feasible to reach, about 10 million volts for protons, 15 million volts for deuterons and $\alpha$-particles. Even these limits have not yet been quite attained.

The possibilities of the cyclotron are so outstanding that, in spite of the expense and labour of construction, many machines have been or are being built in laboratories all over the world. In Great Britain there are two cyclotrons nearly ready for use, one in Cambridge and the other in Liverpool. It may be of interest to give a few details of the construction of the Liverpool cyclotron, although it is not yet in operation.

The electrodes between which the ions are accelerated are two short, hollow semi-cylinders of large diameter, about 2 inches high and about 
30 inches diameter, made of copper sheet. These, known as the $D$ plates, are contained in a large chamber or tank. Hydrogen (for a proton beam), or heavy hydrogen (for deuterons) is admitted to the tank, so that a pressure of about $10^{-4} \mathrm{~mm}$. is maintained.

The ions are produced at the centre of the chamber, between the $D$ electrodes, by bombarding the gas with an intense stream of electrons from a heated filament.

The alternating voltage is applied to the $D$ 's by coupling them to an oscillating circuit. The generator of the oscillations consists of two large triode valves coupled in push-pull, using a selfoscillating circuit. It is important to make the voltage difference between the $D$ 's as high as possible, and therefore the oscillation generator must be able to supply a considerable amount of power, certainly more than $20 \mathrm{kw}$. The voltage between the $D$ 's in Lawrence's experiments is about 45,000 volts.

The vacuum chamber, containing the $D$ plates, etc., is placed between the poles of an electromagnet. As it is important, in order to obtain resonance, that the field should be uniform over the whole path of the ions, the diameter of the poles must be sensibly greater than that of the $D$ plates. The magnet must therefore be very large, and a fair amount of power will be required to maintain the magnetic field. Our Liverpool magnet contains 46 tons of iron and 8 tons of copper. (The copper was generously presented to me by the directors of British Insulated Cables, Ltd.). The diameter of the pole faces is about 36 inches and the air gap between them is 8 inches. The power consumption in normal running conditions will be from 40 to $50 \mathrm{kw}$., under full load about $70 \mathrm{kw}$. The maximum field under the conditions of experiment, that is, with a working gap of 5 inches, is about 19,000 gauss.

When the ions come to the periphery of one of the $D$ 's, they are allowed to pass through an opening where the $D$ is cut away. An electric field is applied between a plate-the deflector plate-and the $D$ to deflect them from their circular path towards a window in the tank. The ions can thus be brought out of the tank and made more readily available for experiment.

The intensity of the ion beam which is obtained in this way depends of course on the number of ions produced initially at the centre of the chamber. It depends also on a very careful adjustment of the magnetic field, by means of which the ions are concentrated near the median plane of the $D$ plate. In Lawrence's early experiments the ion current was of the order of 0.01 microampere. Now, by improvement of the conditions, Lawrence can maintain an ion current of 150 micro- amperes for many hours. The number of particles carried by such a current is $10^{15}$ per second, equal to the number of $\alpha$-particles emitted by $30 \mathrm{kgm}$. of radium. Moreover, the energy of the particles is greater-about 8 million volts-nearly double the energy of the fastest $\alpha$-particle from radioactive bodies.

\section{The Applications}

When the stream of fast particles-protons, deuterons, or $\alpha$-particles-issuing from the cyclotron is allowed to fall on a target of an element, some of the atomic nuclei may undergo transmutation. The incident particle enters the nucleus and a new nucleus is formed which disintegrates with the emission of a different particle. In many of these transmutations, neutrons are emitted, and these in their turn can be used as projectiles for the transmutation of elements.

The general processes of nuclear transmutation are well known and need not be described in detail here. It will be sufficient to note that more than four hundred nuclear reactions of this primary type have already been discovered. Many new forms of atomic nuclei have been produced in these reactions. Nearly all these new atomic nuclei are isotopes of the ordinary chemical elements. They differ from the known isotopes in that they are unstable and transform, in the course of time, with the emission of a negative or positive electron into a stable nucleus. This is the phenomenon of artificial radioactivity discovered by Mme. and M. Curie-Joliot. Such changes as these may be called secondary nuclear reactions. About two hundred or more cases of this type are known, some of which are of special interest.

In general terms, one may say that the physical applications are directed to the study of atomic nuclei, their transmutation by bombardment with different nuclei, the investigation of the properties of neutrons and of the interaction of fast particles with matter.

I should like, however, to refer to certain applications of the cyclotron which depend on these nuclear reactions, and in particular to the biological applications.

One may draw an analogy between the cyclotron and the X-ray tube. In the X-ray tube the stream of electrons is used to produce X-rays ; the fast ions issuing from the cyclotron can be used, by bombarding beryllium for example, to produce a stream of neutrons. When we remember the uses of X-rays and of radium in the treatment of malignant tissues, it is natural to ask what are the possible applications of neutrons.

The ionizing effects of neutrons are rather different from those of X-rays. The X-rays impart their energy to the electrons of matter 
through which they pass, and these secondary electrons produce relatively small ionization over a long path. Neutrons do not lose energy in collision with electrons but in collision with the nuclei of atoms. In passing through living matter, neutrons will lose energy mainly in colliding with the hydrogen atoms, producing recoil protons which ionize very strongly over a short path. For example, 2 M.e.v. neutrons passing through tissues will frequently produce recoil protons of about 300,000 volts energy. These will produce about 10,000 ions in a distance of some 4-5 $\mu$. A secondary electron produced by X-rays would give less than 100 ions in the same path.

It seems that biological effects on individual cells depend more on ionization density than on the total number of ions, and therefore one might expect that neutrons will be biologically more effective than $\mathrm{X}$-rays giving the same total ionization. There is a certain amount of evidence which indicates that this is indeed the case, but the results are too meagre to permit a definite conclusion. For a given ionization, neutrons appear to be about five times as effective as X-rays in destroying malignant cells or in changing the blood picture of the rat, and about ten times as effective in retarding the growth of wheat seedlings. Many experiments are now in progress both on plant and on animal tissues.

By means of neutron irradiation it may be possible to produce biological effects in specific regions. There are some elements which show strong absorption of slow neutrons, for example, lithium and boron. Ordinarily, animal tissues do not contain any significant amount of these elements, but it may prove possible to inject into the tissues in a suitable form compounds of such elements. Irradiation of the tissues with slow neutrons would then cause the release of considerable energy in the small volume containing the injected substance.

\section{RADIOACTIVE INDICATORS}

Another aspect of the application of the cyclotron to biological investigations arises from its use in the production of artificial radioactive elements. Nearly all the ordinary chemical elements can now be obtained in radioactive modifications. These have exactly the same chemical properties as the usual forms. The radioactive isotope will accompany its inactive isotope through any series of chemical processes, however complicated these may be; but the active isotope can always be recognized by its radioactivity, which acts as a label enabling us to detect the presence of a particular group of atoms and to follow this group throughout the chemical processes. Thus, if we wish to study the distribution of a certain element in a series of biological processes, we can mix with it a radioactive isotope, and we can follow the distribution by means of the radioactivity.

Up to the present, the use of active indicators in biological investigations has been almost entirely confined to the role of phosphorus in metabolism. Apart from its structural function along with calcium as a major constituent of bones and teeth, phosphorus, as phosphoric acid, plays a predominant part in the intermediate metabolism of a variety of substances. An active modification of phosphorus can be produced in different ways, one of which is the deuteron bombardment of phosphorus :

$$
\begin{gathered}
{ }_{15}^{31} \mathrm{P}+{ }_{1}^{2} \mathrm{H} \rightarrow{ }_{15}^{32} \mathrm{P}+{ }_{1}^{1} \mathrm{H} \\
{ }_{15}^{32} \mathrm{P} \rightarrow{ }_{16}^{32} \mathrm{~S}+{ }_{-1}^{0} e
\end{gathered}
$$

The radio-phosphorus breaks up with the emission of a $\beta$-particle and the formation of ${ }^{32} \mathrm{~S}$. It decays to half value in $14 \frac{1}{2}$ days, a very convenient period.

Radio-phosphorus was first used as an indicator by Chiewitz and Hevesy in the study of phosphorus metabolism in rats. The active phosphorus is mixed with inactive phosphorus and converted into sodium phosphate. This can be mixed with the food of the rat. The fate of the phosphoruswhether excretion, deposition, transfer from one tissue to another, and the biological synthesis of compounds which contain phosphorus-can then be followed by tracing the radioactive atoms.

In this way it was found that a large part of the phosphorus was incorporated in various compounds in the bones and muscles, from which it is gradually displaced. The experiments confirm the idea that the mineral matter of bone is in a dynamic state, in which the bones are continually losing phosphorus atoms and taking up others which are later, in their turn, replaced.

Another example of the use of phosphorus is given by an experiment of Hahn and Hevesy. It is generally assumed that no regeneration of the brain tissue of adult animals takes place. Hahn and Hevesy found, however, that one hour after a subcutaneous injection of labelled sodium phosphate, labelled lecithin was already formed in the brain tissue of fully grown rats. Their experiments suggest that a constant breakdown and building up of lecithin takes place in the brain tissue.

These examples are sufficient to show that by the use of radio-phosphorus as an indicator it is possible to provide a ready answer to such questions as: How much of the phosphorus taken in by the body at a given moment reaches the bones or teeth? How long does it take to arrive there? 
By what path does it arrive? How long does it stay? How does the distribution of phosphorus depend on abnormal conditions of diet or disease ?

Investigations such as these are now beginning in various laboratories. They will not be restricted to the study of phosphorus, for other elements of interest in bodily metabolism such as calcium, potassium, iron, etc., are available in active modifications. I think it is clear that this method of radioactive indicators has many interesting possibilities, for its power and delicacy make it possible to attack problems which have so far been inaccessible to experiment.
There are also other applications of the radioactive modifications of suitable elements. If an element is concentrated in certain organs, then these organs can be subjected to selective irradiation by using an active isotope of the element. For example, radio-phosphorus can be used to irradiate bone and bone marrow, or spleen; radioiodine for thyroid, etc. So little work has been done on these lines that it would be premature to discuss these therapeutic applications further. They may, however, prove to be important and among the most spectacular results of experiments on artificial transmutation.

\section{History of the Fenland*}

$\mathrm{W}$ ITHIN historic times, the English fenland stretched over the greater part of the area to the west and south of the Wash, extending as far north as Lincoln and as far south as Huntingdon and Cambridge. On the seaward side, the surface deposits are semi-marine silts, laid down, and afterwards occupied, during the RomanoBritish period. On the landward side, the upper layers are peat produced by discharge of the flood waters of the Rivers Witham, Welland, Nene and Ouse into the extensive shallow basin of the fens. The fen peats are alkaline and therefore support a vegetation of the true 'fen' type. Very little of the original vegetation of the peat fen remains, however, since the whole area has been drained and brought under extensive cultivation. Its present characteristics are the black peaty soil, uniform flatness and deep ditches full of reeds (Phragmites communis) which separate fields of cereals, potatoes and sugar beet.

Cambridge is at the head of the fens, and the town and University have become the centre of the activities of the Fenland Research Committee, formed in 1932 under the presidency of Sir Albert Seward, and aided by grants from - the British Association, the Percy Sladen Trust and the Department of Scientific and Industrial Research. Thus have the geology, botany, archæology, stratigraphy and climate of the fenland area been closely investigated. One fen area which has yielded exceptionally valuable results is Wicken Fen, the largest area still uncultivated, covering about one square mile, now in the hands of the National Trust, and lying about ten miles to the north-east of Cambridge on the margin of the fenland itself.

\footnotetext{
* Based on the evening discourse by Dr. H. Godwin to the British
} Association at Cambridge on August 19.
Very little is known of the fenland in glacial times; for most of our knowledge of the area we must turn to the post-glacial period, during which the most important single key to its history lies in the recognition of the land- and sea-level movements which have left their record in the deposits of the basin.

The upper layers are very similar to those of the German coastal marshes. Although the peat is continuous at the margin, it is soon separated into an upper and lower layer by wedging out of soft grey clay. The lower layer contains brush. wood of oak, pine and alder; the upper layer contains some tree remains and above this a layer of Sphagnum peat of a type similar to that of the large red bogs of the central Irish plain. In certain parts there is a layer of calcareous shell-marl overlying the Sphagnum peat. This reflects very changed conditions, since Sphagnum could not have grown in water so deep or so calcareous. From investigations of the diatoms and foraminifera of the fen clay, it is concluded that it was deposited in brackish water and that there was also considerable marine influence. This shows that a phase of freshwater peat formation with fen woods was interrupted by a marine transgression, and that, after the upper peat had formed, a phase of increased wetness produced shallow lakes.

In places nearer the sea, there is upper silt several feet thick and several feet above sea-level. Its foraminifera content shows it to represent another marine phase. The upper peat extends below it at about ordnance datum for many miles seawards. The surface of this silt shows abundant traces of Romano-British occupation. In parts, the upper peat has been worn away by drainage and cultivation, exposing the surface of the fen clay at ordnance datum. Thus evidence is found 